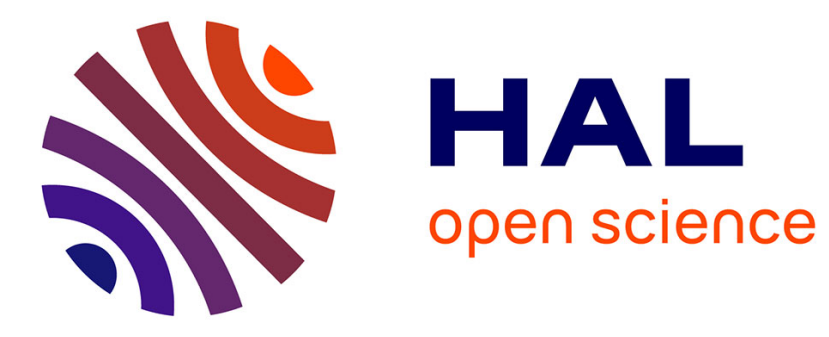

\title{
Shape part Transfer via semantic latent space factorization
}

\author{
Raphaël Groscot, Laurent D. Cohen, Leonidas Guibas
}

\section{To cite this version:}

Raphaël Groscot, Laurent D. Cohen, Leonidas Guibas. Shape part Transfer via semantic latent space factorization. Geometric Science of Information (GSI2019), Aug 2019, Toulouse, France. pp.511-519, 10.1007/978-3-030-26980-7_53. hal-02281621

\section{HAL Id: hal-02281621 \\ https://hal.science/hal-02281621}

Submitted on 16 Dec 2020

HAL is a multi-disciplinary open access archive for the deposit and dissemination of scientific research documents, whether they are published or not. The documents may come from teaching and research institutions in France or abroad, or from public or private research centers.
L'archive ouverte pluridisciplinaire HAL, est destinée au dépôt et à la diffusion de documents scientifiques de niveau recherche, publiés ou non, émanant des établissements d'enseignement et de recherche français ou étrangers, des laboratoires publics ou privés. 


\title{
Shape part transfer via semantic latent space factorization
}

\author{
Raphaël Groscot ${ }^{1 \star}$, Laurent Cohen ${ }^{1}$, and Leonidas Guibas ${ }^{2}$ \\ 1 University Paris Dauphine, PSL Research University \\ CEREMADE, CNRS, UMR 7534, 75016 Paris, France \\ 2 Stanford University
}

\begin{abstract}
We present a latent space factorization that controls a generative neural network for shapes in a semantic way. Our method uses the segmentation data present in a shapes collection to explicitly factorize the encoder of a pointcloud autoencoder network, replacing it by several sub-encoders. This allows to learn a semantically-structured latent space in which we can uncover statistical modes corresponding to semantically similar shapes, as well as mixing parts from several objects to create hybrids and quickly exploring design ideas through varying shape combinations. Our work differs from existing methods in two ways: first, it proves the usefulness of neural networks to achieve shape combinations and second, adapts the whole geometry of the object to accommodate for its different parts.
\end{abstract}

Keywords: autoencoder $\cdot$ pointcloud $\cdot$ latent space

\section{Introduction}

Design ideas exploration is a necessary step for creative modeling. Building tools that help quickly prototyping ideas can significantly improve designers' workflow. Given the tremendous size of 3D shape repositories, scanning all previously existing models can be cumbersome. This is why we propose, in this work, a first step to building such a tool: a shape composer that allows to combine parts coming from different objects into a single and coherent new object. Unlike other works that extract and snap different parts into new positions, we explore the possibility of holistic composition with the use of generative neural networks.

This paper presents a semantically-rich way of controlling generative networks for $3 \mathrm{D}$ shapes, without limiting the user to predefined labels. On the contrary, our approach is essentially data-driven in two ways. First, because we rely on a large collection of shapes to train our generative model; second, because the dataset itself is used by the user to tweak the output. More specifically, the dataset contains various shapes along with their segmentations into meaningful object parts. Our generative network is then trained to produce shapes in a way

\footnotetext{
* This work was initiated during a long visit in the Geometric Computation group at Stanford University
} 
that is compatible with the segmentation. This is achieved by factorizing the latent space of the generative model according to the different possible shape parts. Thanks to this, a user can edit any given shape and decide to only change part of it, by picking the desired geometry within the dataset. Moreover, the network automatically adapts the final shape in a holistic way to make sure the new part fits naturally.

\section{Related work}

Our method is related to different research efforts in 3D shapes analysis and generation. We separate our review in three categories: generative modeling, shapes neural networks, and data-driven shapes editing.

Generative neural networks Generative models suchs as GANs [6] and VAEs [10] both offer ways to sample from a distribution that matches a given dataset. VAEs rely on an autoencoder scheme, where a network computes a lower dimensionality code that represents a sample from the data and recreates it. Adding a variational constraint that imposes a prior (e.g. gaussian) on the latent distribution makes sure that the model generalizes well. Their compression-like behavior can then be used for several tasks among which unsupervised learning, sampling, interpolation and denoising [4]. One drawback is that the output is typically blurred, because their loss doesn't account for a perceptual term. On the contrary, GANs aim at mimicking a given distribution by generating samples that are indistinguishable from the original dataset; they can hence generate much sharper results, to the cost of harder training and difficulty to control for mode collapse [12]. Conditioning on the likelihood [3, 16] allows to have a finer control on their outputs. Our work aims at the same property by means of imposing a specific factorization on an autoencoder latent space.

Shape neural networks As opposed to images, 3D shapes don't naturally fit in a neural network framework. The main issue is to represent them in a fixedsize euclidian domain. The most direct way to do so is to use voxel grids and directly transpose Convolutional Neural Networks in 3D [7]. However, even if this approach can yield good results, generated shapes quality is limited by the grid discretization and the $O\left(n^{3}\right)$ complexity. To overcome these limits, Pointnet [17] introduced a neural network architecture based on pointclouds and permutationinvariant operators, which characterizes well an unordered set such as a pointcloud. It has successfully shown its usefulness for tasks such as classification and segmentation, and even has an extension that exploits hierarchical analysis [18]. This architecture can also be used to generate pointclouds from photographs [5]. Lastly, [2] has replaced the permutation invariance constraint by imposing a lexicographic order on the pointset, leading to pointcloud GANs with high reconstruction accuracy. Our method relies on a variation of such a shape neural networks, tailored at being used for shape combinations. 
Data-driven shape editing Many existing methods give automated tools for shapes editing and design exploration. Existing works range from shape correspondences $[7]$ to style similarity and transfer $[13,14]$. Others focus on generating diversity, by extracting and snapping parts together [8], or by hierchical shape analysis and synthesis [11]. While [8] creates a combinatorial diversity, our method tries to achieve a geometric diversity. We also share a common usage as [14], but while they use an example to guide the overall style, we use an example to guide the shape a of given part.

\section{Method}

\subsection{Autoencoder foundation}

Our goal is to create new object shapes, by generating variations within their different parts, in a data-driven process. The first step is to be able to recreate objects from the dataset. A natural choice is to use a generative model, we chose autoencoders. Formally, the goal is to learn the two functions $E$ (encoder) and $D$ (decoder) such that, for all $\mathrm{X}$ in the dataset:

$$
X=D(E(X))
$$

These two functions are implemented as neural networks that operate on pointclouds. The key specificity of our method is our factorization of $E$ based on the available segmentation data. The architecture of our foundational autoencoder is the following ( $N=1024$ points):

input: a minibatch of 32 pointclouds, each represented as a $N x 3$ matrix, accompanied by their segmentation data (see part 3.2)

encoder: based on Pointnet [17] but in a much simpler version, with successive layers of per-point filters followed by ReLU layers

code mixer: the latent space factorization step, as explained in part 3.3 decoder: three fully connected layers with biases, except on the last layer output: the last layer is ultimately reshaped to a $N x 3$ matrix

\subsection{Consistent segmentation data}

To demonstrate our method, we use the planes category from ShapenetCore and its segmentation obtained from [19], comprising of the four following parts: body, wings, engine, tail (we restricted our analysis only to models containing the four parts). Since all models are aligned in a consistent manner (the plane body is aligned with the $\mathrm{Z}$ axis), our neural networks doesn't need any rotational invariance, and can leverage from the strong spatial relations of the models' parts for both the encoder and decoder. 


\subsection{Semantic latent space factorization}

We use pointclouds to represent surfaces, a choice that leads to the following remark: any subset of a pointcloud is a pointcloud. Although this may seem trivial, note that this is not a property that usually holds in a machine learning setting: for instance, a segmented region in an image is not typically rectangular. This allows us to replace the encoder by $K$ encoders, each for a part, which yields the following factorization:

$$
\begin{gathered}
E=E_{1} * E_{2} * \ldots * E_{K} \\
E(X)=C=\left[c_{1}, c_{2}, \ldots, c_{n}\right], c_{i}=E_{i}(X)
\end{gathered}
$$

where each $E_{i}$ represents a partial encoder for part i, and evaluates what we call a subcode. The above product corresponds to vector concatenation. In this form, the factorization of the latent space simply corresponds to assigning parts to dedicated coordinates. Figure 1 shows a diagram of the corresponding pipeline.

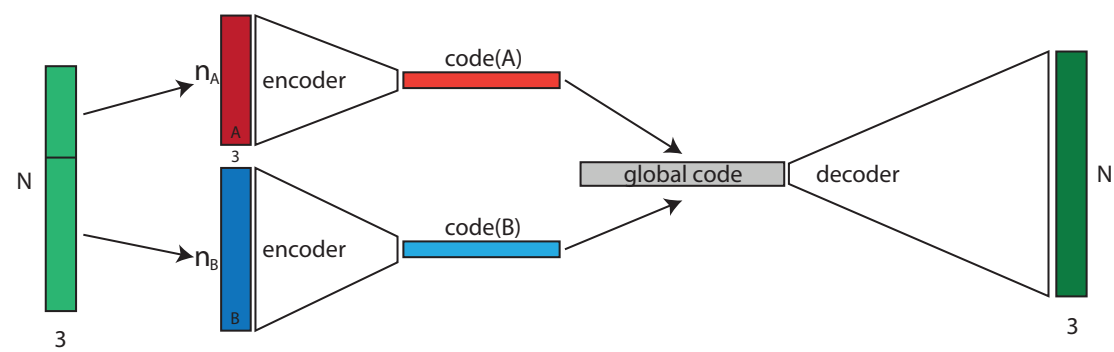

Fig. 1: Structure of our hybrid encoder model, illustrated for simplicity with $K=2$ parts

\subsection{Loss}

When it comes to pointclouds, two reconstruction losses can be considered: Chamfer and Earth Mover's Distance (EMD). We chose the former for its easy implementation; the interested reader will find in [5] a comparison of both losses.

$$
d_{C D}\left(S_{1}, S_{2}\right)=\sum_{x \in S_{1}} \min _{y \in S_{2}}\|x-y\|_{2}^{2}+\sum_{y \in S_{2}} \min _{x \in S_{1}}\|x-y\|_{2}^{2}
$$

\section{Experiments}

We implemented our architecture using Tensorflow [1] and ran it on an Nvidia Gti1080 GPU. We trained over 40 epochs using Adam [9] with learning rate of 0.9 and a batchsize of 32 . 


\subsection{Basic autoencoder mode}

Since our network is based on an autoencoder, we first demonstrate its ability to reconstruct objects from the training set. Figure 2 shows examples of reconstructions, chosen to be representative of the type of objects present in our dataset. We can notice that the reconstruction quality highly depends on the sub category (not available) of the object: the typical plane present in the dataset is similar to the second column, so this is where the autoencoder concentrated most of its capacity.
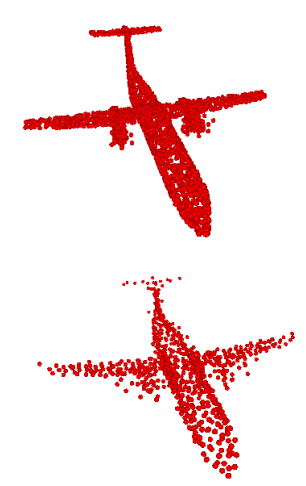
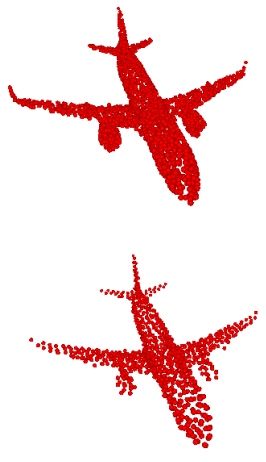
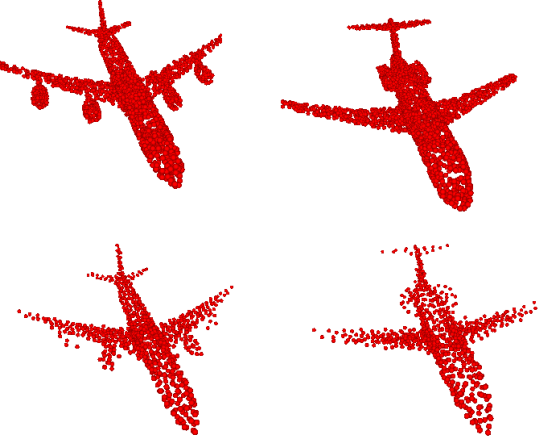

Fig. 2: Example of some reconstructions. Top row: original. Bottom row: reconstructed

Clustering The latent codes computed by $E$ can be explored using standard dimensionality reduction techniques, such as PCA and tSNE [15]. Figure 3 shows the tSNE projection of our latent space over 2 dimensions, and snapshots of certain blobs with their corresponding shapes. Note how similar shapes live in the same blob. As with any tSNE projection, we remind the reader that distances between blobs are not significant.

Continuous part transfer Thanks to the factorization of $E$, by simply interpolating on a given $E_{i}$, we can easily transfer a part of an object to another one while keeping the rest of the object unchanged. Let $S$ be a source object, $T$ the target and $i$ the index of the part we wish to transfer from $S$ to $T$. This is done with $E(T)=E_{1}(T) * E_{2}(T) * \ldots * E_{K}(T)$, replacing $E_{i}(T)$ by $E_{i}(S)$. A linear interpolation between $E_{i}(T)$ and $E_{i}(S)$ effectively realizes the continuous morphing of the part. Figure 4 shows the results of selectively transfering parts of a plane onto another one. 


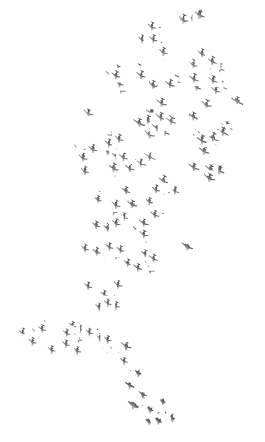

(a) Blob from the left

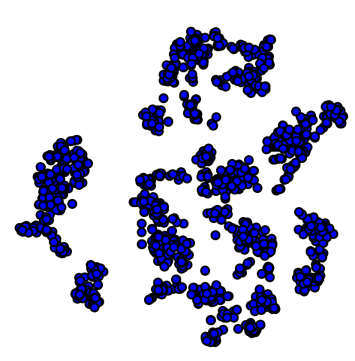

(b) Latent space

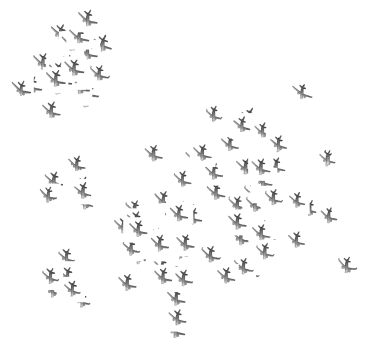

(c) Blob from the middle right

Fig. 3: tSNE projection of the encoder latent space, with close-ups of two blobs

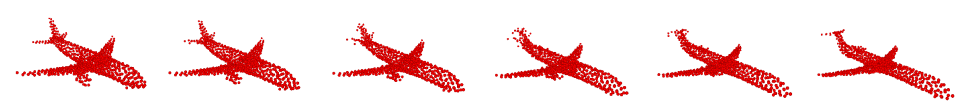

(a) Interpolation of the whole plane

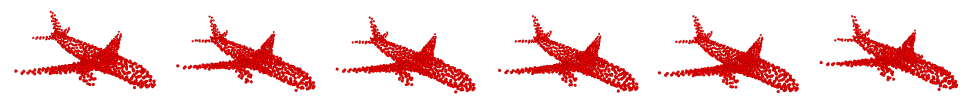

(b) Interpolation only on: wing

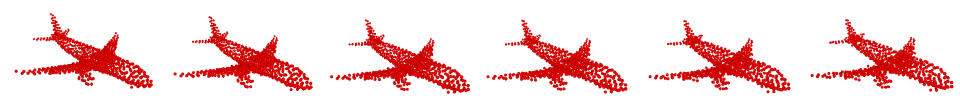

(c) Interpolation only on: body

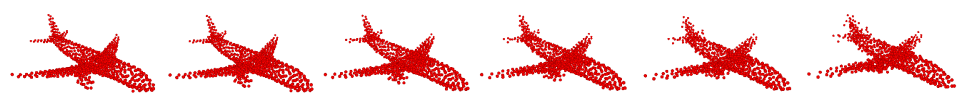

(d) Interpolation only on: engine

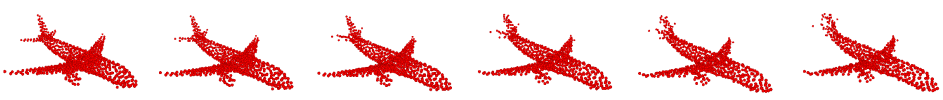

(e) Interpolation only on: tail

Fig. 4: Selective part transfer, compared to the global interpolation from a source to a target plane 


\section{$5 \quad$ Limitations and discussion}

An inherent limitation of our model is that of the autoencoder it is based upon. Indeed, it suffers from a problem slightly similar to mode collapse, as shown in Figure 2: it focuses all its reconstruction capacity towards the most frequent shapes from the dataset, which means that it cannot be suited for part transfer when one part belongs to an atypical object. Another limitation is that small details can be lost, but are a major concern when they belong to a discriminative part. For instance, once can think of a plane with 4 engines: overall, the engines only have a medium contribution to the reconstruction loss. Adopting a partspecific loss could be a way of circumventing this problem.

As for part transfer, our holistic approach has both pros and cons. Since we want the whole model to adapt for the new shape, we do not want to limit the geometry changes to the region of the transfered part. However, in its current state, it is still hard to predict the general evolution of an object upon part transfer.

Acknowledgement. This work was made possible by an exchange program between PSL-ITI and Stanford University. The authors gratefully acknowledge the financial support coming from PSL, Fondation du Judaïsme Français and Fondation Armand et Janet Sibony.

\section{References}

1. Abadi, M., Agarwal, A., Barham, P., Brevdo, E., Chen, Z., Citro, C., Corrado, G.S., Davis, A., Dean, J., Devin, M., Ghemawat, S., Goodfellow, I., Harp, A., Irving, G., Isard, M., Jia, Y., Jozefowicz, R., Kaiser, L., Kudlur, M., Levenberg, J., Mané, D., Monga, R., Moore, S., Murray, D., Olah, C., Schuster, M., Shlens, J., Steiner, B., Sutskever, I., Talwar, K., Tucker, P., Vanhoucke, V., Vasudevan, V., Viégas, F., Vinyals, O., Warden, P., Wattenberg, M., Wicke, M., Yu, Y., Zheng, X.: TensorFlow: Large-scale machine learning on heterogeneous systems (2015), http://tensorflow.org/, software available from tensorflow.org

2. Achlioptas, P., Diamanti, O., Mitliagkas, I., Guibas, L.J.: Learning representations and generative models for $3 \mathrm{D}$ point clouds (2017)

3. Chen, X., Duan, Y., Houthooft, R., Schulman, J., Sutskever, I., Abbeel, P.: Infogan: Interpretable representation learning by information maximizing generative adversarial nets (2016)

4. Doersch, C.: Tutorial on Variational Autoencoders. arXiv e-prints arXiv:1606.05908 (Jun 2016)

5. Fan, H., Su, H., Guibas, L.J.: A point set generation network for 3D object reconstruction from a single image. CoRR abs/1612.00603 (2016), http://arxiv.org/abs/1612.00603

6. Goodfellow, I.J., Pouget-Abadie, J., Mirza, M., Xu, B., Warde-Farley, D., Ozair, S., Courville, A., Bengio, Y.: Generative adversarial networks (2014)

7. van Kaick, O., Zhang, H., Hamarneh, G., Cohen-Or, D.: A survey on shape correspondence. Computer Graphics Forum 30(6), 1681-1707 (2011). https://doi.org/10.1111/j.1467-8659.2011.01884.x, https://onlinelibrary.wiley.com/doi/abs/10.1111/j.1467-8659.2011.01884.x 
8. Kalogerakis, E., Chaudhuri, S., Koller, D., Koltun, V.: A probabilistic model for component-based shape synthesis. ACM Trans. Graph. 31(4), 55:1-55:11 (Jul 2012). https://doi.org/10.1145/2185520.2185551, http://doi.acm.org/10.1145/2185520.2185551

9. Kingma, D.P., Ba, J.: Adam: A Method for Stochastic Optimization. arXiv e-prints arXiv:1412.6980 (Dec 2014)

10. Kingma, D.P., Welling, M.: Auto-Encoding Variational Bayes. arXiv e-prints arXiv:1312.6114 (Dec 2013)

11. Li, J., Xu, K., Chaudhuri, S., Yumer, E., Zhang, H., Guibas, L.J.: GRASS: Generative recursive autoencoders for shape structures. ACM Transactions on Graphics (Proceedings of SIGGRAPH 2017) (2017)

12. Locatello, F., Vincent, D., Tolstikhin, I.O., Rätsch, G., Gelly, S., Schölkopf, B.: Clustering meets implicit generative models. CoRR abs/1804.11130 (2018), http://arxiv.org/abs/1804.11130

13. Lun, Z., Kalogerakis, E., Sheffer, A.: Elements of style: Learning perceptual shape style similarity. ACM Transactions on Graphics 34(4) (2015)

14. Lun, Z., Kalogerakis, E., Wang, R., Sheffer, A.: Functionality preserving shape style transfer. ACM Trans. Graph. 35(6), 209:1-209:14 (Nov 2016). https://doi.org/10.1145/2980179.2980237, http://doi.acm.org/10.1145/2980179.2980237

15. van der Maaten, L., Hinton, G.E.: Visualizing high-dimensional data using t-SNE. Journal of Machine Learning Research 9, 2579-2605 (2008)

16. Mirza, M., Osindero, S.: Conditional Generative Adversarial Nets. arXiv e-prints arXiv:1411.1784 (Nov 2014)

17. Qi, C.R., Su, H., Mo, K., Guibas, L.J.: Pointnet: Deep learning on point sets for 3D classification and segmentation (2016)

18. Qi, C.R., Yi, L., Su, H., Guibas, L.J.: Pointnet++: Deep hierarchical feature learning on point sets in a metric space. CoRR abs/1706.02413 (2017), http://arxiv.org/abs/1706.02413

19. Yi, L., Kim, V.G., Ceylan, D., Shen, I.C., Yan, M., Su, H., Lu, C., Huang, Q., Sheffer, A., Guibas, L.J.: A scalable active framework for region annotation in 3D shape collections. ACM Trans. Graph. 35(6), 210:1-210:12 (Nov 2016). https://doi.org/10.1145/2980179.2980238, http://doi.acm.org/10.1145/2980179.2980238 